\title{
Is anemia in children with sleep disordered breathing actually a consequence of chronic disease?
}

\author{
Fatih Alper Akcan ${ }^{1}$, Yusuf Dündar², Hümeyra Bayram Akcan ${ }^{3}$, Ahmet Uluat ${ }^{4}$, Derya Cebeci', Mehmet Ali \\ Sungur ${ }^{5}$
}

${ }^{1}$ Department of Otorhinolaryngology, Düzce University School of Medicine, Düzce, Turkey

${ }^{2}$ Department of Otorhinolaryngology, Rize State Hospital, Rize, Turkey

${ }^{3}$ Department of Internal Medicine, Atatürk State Hospital, Düzce, Turkey

${ }^{4}$ Department of Otolaryngology, Evliya Çelebi Training and Research Hospital, Kütahya, Turkey

${ }^{5}$ Department of Biostatistics, Düzce University School of Medicine, Düzce, Turkey

\begin{abstract}
Objectives. We aimed to investigate iron metabolism and laboratory findings of anemia in children undergoing adenotonsillectomy for sleep disordered breathing. Methods. Consecutive children undergoing adenoidectomy/adenotonsillectomy for sleep disordered breathing between January 2013 and January 2015 were investigated. Routine preoperative blood tests including blood count and iron studies were analyzed. Subgroup analyses were performed addressing to the severity of sleep disordered breathing, age and duration of symptoms. Results were compared between subgroups and normal values. Results. The study included 171 children. The mean age was $5.44 \pm 2.62$ years. The mean hemoglobin level was $11.87 \pm 1.125 \mathrm{gr} / \mathrm{dL}$ and 24 $(14 \%)$ out of 171 patients had anemia $(<11 \mathrm{gr} / \mathrm{dL})$. The mean ferritin levels were $23.23 \pm 17.27 \mu \mathrm{g} / \mathrm{L}$ and 21.27 $\pm 15.44 \mu \mathrm{g} / \mathrm{L}$ in patients with anemia and non-anemic patients, respectively $(p=0.572)$. Ferritin levels decreased in only $8(33 \%)$ out of 24 patients with anemia. The mean age, body mass index, hematocrit, mean corpuscular volume, unsaturated iron binding capacity and serum iron levels decreased in patients with anemia. The rate of anemia significantly increased in children less than or equal to 3 years of age $(p=0.020)$. There was no significant association between hemoglobin levels and sleep disordered breathing clinical score or duration of symptoms. Conclusion. The association between low iron status and sleep disordered breathing has previously published. Unfortunately, the type of anemia is still unclear. Our results supposed that the anemia of chronic disease secondary to chronic inflammatory process might play a role in pathogenesis of anemia in patients with sleep disordered breathing.
\end{abstract}

Eur Res J 2018;4(3):220-226

Keywords: Sleep disordered breathing, adenotonsillectomy, anemia, iron deficiency, anemia of chronic disease 


\section{Introduction}

Sleep disordered breathing (SDB) is one of the most common childhood disorder with the range from simple snoring to obstructive sleep apnea (OSA) [1]. Approximately $2-3 \%$ of children are affected with SDB which may lead to behavioral, cognitive and growth abnormalities $[1,2]$. The most common cause of SDB in childhood is upper airway obstruction due to adenotonsillar hypertrophy. Many hypotheses were promoted to clarify pathogenesis of adenotonsillar hypertrophy such as: allergic hypothesis, disordered lymhphoproliferation and biofilm models [3, 4]. Unfortunately, there is no enough data to explain pathogenesis of adenotonsillar hypertrophy despite these hypotheses.

SDB is associated with many co-morbid diseases which is one of the current research topic. SDB might have an impact on iron metabolism. Healthy iron metabolism has a critical importance for normal cognitive and motor development in infancy $[5,6]$. Furthermore, healthy iron metabolism is essential for immune system [7, 8]. Zilberman et al. [9] has published the first paper addressing to association of SDB and impaired iron metabolism. They showed the iron deficiency with SDB in chronic heart failure patients. The other researchers support this finding. The improvement of day-time sleeping in patients with SDB has been published after correcting iron status and anemia [10].

The literature is indicating the increased incidence of iron deficiency in patients undergoing adenotonsillectomy [3, 11, 12]. Unfortunately, these studies mostly included patients operated for recurrent infections. Everland et al. [11] reported anemia rate of $56.3 \%$ in patients undergoing adenotonsillectomy because of recurrent infection and upper airway obstruction. Kerstein et al. [13] evaluated the iron status of children diagnosed with SDB undergoing adenotonsillectomy. They reported low iron status in particular children under 6 years of age. This study concluded the low impaired iron status as an iron deficiency anemia. However, this impaired iron metabolism might be associated with chronic inflammatory process accompanied to SDB. The literature is supporting the increased pro-inflammatory cytokines (IL-6, THF- $\alpha, \mathrm{C}$ reactive protein) and decreases IL-10 which is an anti-inflammatory cytokine [14-17]. Iron deficiency anemia is associated with low hemoglobin $(\mathrm{Hb})$, hematocrit (Hct), mean corpuscular volume (MCV), serum iron level and serum ferritin levels. But, anemia of chronic disease is associated with low serum iron, iron binding capacity and normal or high ferritin levels [18]. Ferritin is a positive acute phase reactant that explains the normal or high ferritin levels in anemia of chronic disease. Everland et al. [11] reported normal ferritin levels in $57 \%$ of children with anemia that is not surprising.

We analyzed the iron metabolism and effects of SDB in patients undergoing adenotonsillectomy for upper airway obstruction.

\section{Methods}

A retrospective clinical trial was conducted in an academic tertiary referral center between January 2013 and January 2015 after obtaining local Medical Ethics Committee approval. A total of 186 adenoidectomy, tonsillectomy and adenotonsillectomy case files were reviewed retrospectively and subjects were included in the study if they operated for SDB. Patients were excluded from the study if they met any of following: 1) nasal septal deviation, 2) history of recurrent tonsillitis, 3) history of anemia treatment in the past 3 months, 4) the other causes of anemia; recurrent epistaxis, bleeding disorders, surgical operations in the past 3 months, 5) history of chronic diseases.

We use a standard questionnaire for SDB preoperatively that include; mouth breathing during sleep, daytime mouth breathing, apnea, snoring, cough, rhinorrhea and restless sleep. Scoring is used for each of above; 0: never, 1: occasionally, 2: frequently and 3: always [19, 20]. Patients were categorized into 3 subgroups according to these questionnaire score; mild: 1-7, moderate: $8-14$ and severe: 15-21. Age groups were conducted as $\leq 3$ years, $>3$ years $-\leq 6$ years and $>6$ years of age. Duration of primary symptoms were categorized as $\leq$ 12 months, $>12$ months $-\leq 24$ months and $>24$ months.

All patients were analyzed for $\mathrm{Hb}$, Hct, $\mathrm{MCV}$, ferritin, unsaturated iron binding capacity, serum iron, aPTT and INR. Results were compared to the World Health Organizations (WHO) normal range.

\section{Statistical Analysis}

Continuous data were summarized as mean \pm standard deviation and categorical data were presented as frequency and percent. Distribution of continuous variables were controlled with Shapiro-Wilks test. 
Comparisons for continuous variables were done using independent samples $t$ test for two groups and one-way ANOVA followed by Tukey HSD post hoc test for three and more groups. Categorical data were analyzed by Pearson chi-square or Fisher's exact tests. Statistical analyses were performed with SPSS v.23 statistical package and statistical significance level was considered as $\alpha=0.05$.

\section{Results}

One hundred and eighty patients undergoing adenoidectomy, tonsillectomy, adenotonsillectomy for SDB were analyzed. The study included 171 patients with sufficient laboratory data. The mean age was 5.44 \pm 2.62 years. Tonsilla platina size was assessed by using the Brodsky grading scale [21]. The adenoid tissue volume was graded from 1 to 4 with flexible endoscopy findings depending on choanal opening [22].

The mean $\mathrm{Hb}$ level was $11.87 \pm 1.125 \mathrm{gr} / \mathrm{dL}$ and
$24(14 \%)$ out of 171 patients had anemia $(\mathrm{Hb}<11$ $\mathrm{gr} / \mathrm{dL})$. The mean ferritin level was $22.28 \pm 17.16$ $\mu \mathrm{g} / \mathrm{L}$. Forty-seven $(27 \%)$ patients had low ferritin levels $(<16 \mu \mathrm{g} / \mathrm{L})$. The mean age $(p<0.001)$, BMI $(p$ $=0.021), \operatorname{Hct}(p<0.001), \operatorname{MCV}(p=0.013)$ and serum iron $(p=0.038)$ levels was lower in patients with anemia. Unsaturated iron binding capacity level was lower in anemia patients but the difference was not statistically significant. Table 1 summarizes the laboratory findings in patients with anemia and normal $\mathrm{Hb}$ levels. Ferritin levels were decreased in only 8 $(33 \%)$ patients with anemia. Furthermore, the mean ferritin levels were $23.23 \pm 17.27 \mu \mathrm{g} / \mathrm{L}$ and $21.27 \pm$ $15.44 \mu \mathrm{g} / \mathrm{L}$ in patients with anemia and non-anemic patients respectively. The increased ferritin levels in patients with anemia documented but the difference was not statistical significant (Table 1).

Table 2 summarizes the demographics according to SDB severity. There were $7(3 \%)$ patients in mild SDB, 91 (49\%) patients in moderate SDB and 88 (48\%) patients in severe SDB groups. The mean ages were $5.86 \pm 1.49$ years, $5.91 \pm 2.77$ years and $4.93 \pm$

Table 1. Comparison of anemic and non-anemic patients

\begin{tabular}{lccc}
\hline & $\begin{array}{c}\text { Hb }<\mathbf{1 1} \mathbf{~ g / d L} \\
(\mathbf{n}=\mathbf{2 4})\end{array}$ & $\begin{array}{c}\text { Hb } \geq \mathbf{1 1} \mathbf{g} / \mathbf{d L} \\
(\mathbf{n}=\mathbf{1 4 7})\end{array}$ & $\boldsymbol{p}$ \\
\hline BMI & $15.96 \pm 1.74$ & $17.04 \pm 2.77$ & $\mathbf{0 . 0 2 1}$ \\
Mean age (year) & $4.00 \pm 1.84$ & $5.69 \pm 2.68$ & $<\mathbf{0 . 0 0 1}$ \\
Ferritin $\boldsymbol{\mu g} / \mathbf{L}$ & $23.23 \pm 17.27$ & $21.27 \pm 15.44$ & 0.572 \\
Hct $(\%)$ & $33.12 \pm 4.46$ & $38.45 \pm 2.17$ & $<\mathbf{0 . 0 0 1}$ \\
MCV (fL) & $74.25 \pm 10.33$ & $79.94 \pm 3.75$ & $\mathbf{0 . 0 1 3}$ \\
UIBC $(\boldsymbol{\mu m o l} / \mathbf{L})$ & $236.27 \pm 90.04$ & $259.82 \pm 64.79$ & 0.145 \\
SI $(\boldsymbol{\mu m o l} / \mathbf{L})$ & $53.59 \pm 29.13$ & $67.39 \pm 28.20$ & $\mathbf{0 . 0 3 8}$ \\
\hline
\end{tabular}

$\mathrm{BMI}=$ body mass index, $\mathrm{Hb}=$ hemoglobin, $\mathrm{Hct}=$ hematocrit, $\mathrm{MCV}=$ mean corpuscular volume, $\mathrm{SI}=$ serum iron, $\mathrm{UIBC}=$ unsaturated iron binding capacity

Table 2. Demographics according to severity of SDB

\begin{tabular}{lccccc}
\hline & $\begin{array}{c}\text { Mild (0-7) } \\
(\mathbf{n}=\mathbf{7})\end{array}$ & $\begin{array}{c}\text { Moderate (8-14) } \\
(\mathbf{n = 9 1 )}\end{array}$ & $\begin{array}{c}\text { Severe (15-21) } \\
(\mathbf{n = 8 8})\end{array}$ & $\boldsymbol{p}$ & General \\
\hline Mean age (year) & $5.86 \pm 1.49$ & $5.91 \pm 2.77$ & $4.93 \pm 2.44$ & $\mathbf{0 . 0 3 8}$ & $5.44 \pm 2.62$ \\
$\quad \leq \mathbf{3}$ years & $0(0.0 \%)$ & $19(20.9 \%)$ & $30(34.1 \%)$ & & $49(26.3 \%)$ \\
$\quad>\mathbf{3}$ - $\leq$ 6 years & $6(85.7 \%)$ & $34(37.4 \%)$ & $37(42.0 \%)$ & $\mathbf{0 . 0 1 3}$ & $77(41.4 \%)$ \\
$\quad>$ 6 years & $1(14.3 \%)$ & $38(41.8 \%)$ & $21(23.9 \%)$ & & $60(32.3 \%)$ \\
Female & $5(71.4 \%)$ & $44(48.4 \%)$ & $44(50.0 \%)$ & 0.570 & $93(50.0 \%)$ \\
Male & $2(28.6 \%)$ & $47(51.6 \%)$ & $44(50.0 \%)$ & & $93(50.0 \%)$ \\
Duration of & $14.29 \pm 9.98$ & $25.15 \pm 17.97$ & $23.76 \pm 14.90$ & 0.233 & $24.09 \pm 16.40$ \\
symptoms & & & & &
\end{tabular}

$\mathrm{SDB}=$ sleep disordered breathing 
Table 3. Laboratory findings in comparison with severity of SDB

\begin{tabular}{lcccc}
\hline & $\begin{array}{c}\text { Mild (0-7) } \\
(\mathbf{n}=\mathbf{7})\end{array}$ & $\begin{array}{c}\text { Moderate (8-14) } \\
(\mathbf{n}=\mathbf{8 4})\end{array}$ & $\begin{array}{c}\text { Severe (15-21) } \\
(\mathbf{n}=\mathbf{8 0})\end{array}$ & $\boldsymbol{p}$ \\
\hline $\mathbf{H b}<\mathbf{1 1} \mathbf{~ g / d L}$ & $0(0.0 \%)$ & $13(15.7 \%)$ & $11(13.6 \%)$ & 0.524 \\
Ferritin < 16 $\boldsymbol{\mu g} / \mathbf{L}$ & $1(16.7 \%)$ & $23(27.3 \%)$ & $23(28.75 \%)$ & 0.713 \\
Hct $(\%)$ & $37.04 \pm 1.85$ & $37.86 \pm 3.86$ & $37.59 \pm 2.46$ & 0.736 \\
MCV (fL) & $82.16 \pm 2.41$ & $78.96 \pm 6.42$ & $79.07 \pm 4.62$ & 0.337 \\
UIBC $(\boldsymbol{\mu m o l} / \mathbf{L})$ & $216.00 \pm 65.61$ & $250.75 \pm 62.76$ & $263.64 \pm 75.98$ & 0.239 \\
SI $(\boldsymbol{\mu m o l} / \mathbf{L})$ & $82.60 \pm 50.30$ & $71.31 \pm 27.88$ & $57.78 \pm 24.92$ & $\mathbf{0 . 0 0 7}$ \\
\hline
\end{tabular}

$\mathrm{Hb}=$ hemoglobin, $\mathrm{Hct}=$ hematocrit, $\mathrm{MCV}=$ mean corpuscular volume, $\mathrm{SDB}=$ sleep disordered breathing, $\mathrm{SI}=$ serum iron, $\mathrm{UIBC}=$ unsaturated iron binding capacity

Table 4. Laboratory findings in comparison with age groups

\begin{tabular}{|c|c|c|c|c|}
\hline & $\begin{array}{c}\leq 3 \text { years } \\
(n=41)\end{array}$ & $\begin{array}{c}>3-\leq 6 \text { years } \\
(n=76)\end{array}$ & $\begin{array}{c}>6 \text { years } \\
(\mathrm{n}=54)\end{array}$ & $p$ \\
\hline $\mathrm{Hb}<11 \mathrm{~g} / \mathrm{dL}$ & $11(26.8 \%)$ & $9(11.8 \%)$ & $4(7.4 \%)$ & 0.020 \\
\hline Ferritin $<16 \mu \mathrm{g} / \mathrm{L}$ & $14(34.1 \%)$ & $27(35.1 \%)$ & $6(10.9 \%)$ & 0.005 \\
\hline Het $(\%)$ & $35.88 \pm 4.29$ & $37.74 \pm 2.60$ & $39.02 \pm 2.24$ & $<0.001$ \\
\hline MCV (fL) & $76.72 \pm 8.15$ & $79.36 \pm 3.46$ & $80.68 \pm 4.86$ & 0.002 \\
\hline UIBC $(\mu \mathrm{mol} / \mathrm{L})$ & $260.28 \pm 70.32$ & $258.22 \pm 70.77$ & $247.40 \pm 67.02$ & 0.630 \\
\hline SI $(\mu \mathrm{mol} / \mathrm{L})$ & $58.23 \pm 23.31$ & $67.49 \pm 30.75$ & $69.77 \pm 28.24$ & 0.139 \\
\hline
\end{tabular}

$\mathrm{Hb}=$ hemoglobin, $\mathrm{Hct}=$ hematocrit, $\mathrm{MCV}=$ mean corpuscular volume, $\mathrm{SI}=$ serum iron, $\mathrm{UIBC}=$ unsaturated iron binding capacity

2.44 years in the mild, moderate and severe SDB groups, respectively $(p=0.038)$. The mean age in the severe group was significantly lower than in the mild and moderate group. All patients with anemia were in moderate and severe SDB groups but $\mathrm{Hb}, \mathrm{Hct}, \mathrm{MCV}$, ferritin, and unsaturated iron binding capacity were not significantly different in these subgroups (Table $3)$. Serum iron was significantly difference among SDB groups $(p=0.007)$. When we categorize the patients depending on apnea score, as low apnea score ( 0 or 1 ) and high apnea score ( 2 or 3 ), there were 96 patients with low apnea score and 75 patients with high apnea score. The anemia rates $(\mathrm{Hb}<11 \mathrm{~g} / \mathrm{dL})$ between the low and high apnea score groups were not different, $11.5 \%$ and $17.3 \%$, respectively $(p=0.272)$. However, lower ferritin rate (Ferritin $<16 \mu \mathrm{g} / \mathrm{L}$ ) was $20.0 \%$ in low apnea score group and $37.0 \%$ in high apnea score group, and the difference was statistically significant $(p=0.013)$. The other questionnaires were not significantly associated with anemia when analyzed each of them.

Table 4 summarizes the subgroups according to age. $\mathrm{Hb}, \mathrm{Hct}, \mathrm{MCV}$ and ferritin levels were significantly decreased in small age group $(\leq 3$ years of age). The anemia rates $(\mathrm{Hb}<11 \mathrm{~g} / \mathrm{dL})$ between the $\leq 3$ years of age and $>6$ years of age groups were different, while the $>3-\leq 6$ years of age group was similar to them. The lower ferritin rates (Ferritin $<16$ $\mu \mathrm{g} / \mathrm{L})$ in the $\leq 3$ years of age and $>3-\leq 6$ years of age groups were higher than in the $>6$ years of age group. The mean of Hct rates were different in all groups while MCV level in small age group was significantly lower than in the other age groups. There was no significant association for $\mathrm{Hb}, \mathrm{MCV}$, serum iron, unsaturated iron binding capacity level and ferritin levels according to duration of symptoms (Table 5). Hct levels were significantly increased in patients with extended duration of symptoms $(p=0.042)$.

\section{Discussion}

SDB is a common health problem in children with the estimated incidence of $2-3 \%$. SDB may lead to numerous morbidities such as neurocognitive disorders, craniofacial growth abnormalities, enuresis and cardiovascular problems if not treated [1]. The 
Table 5. Laboratory findings in comparison with duration of symptoms

\begin{tabular}{lcccc}
\hline & $\begin{array}{c}<\mathbf{1 2} \text { months } \\
(\mathbf{n}=\mathbf{7 2})\end{array}$ & $\begin{array}{c}\mathbf{1 2 - 2 4} \text { months } \\
(\mathbf{n}=\mathbf{4 9})\end{array}$ & $\begin{array}{c}>\mathbf{2 4} \text { months } \\
(\mathbf{n}=\mathbf{5 0})\end{array}$ & $\boldsymbol{p}$ \\
\hline $\mathbf{H b}<\mathbf{1 1} \mathbf{~ g / d L}$ & $10(13.9 \%)$ & $9(18.4 \%)$ & $5(10.0 \%)$ & 0.487 \\
Ferritin $<\mathbf{1 6} \boldsymbol{\mu g} / \mathbf{L}$ & $17(23.0 \%)$ & $18(37.5 \%)$ & $12(23.5 \%)$ & 0.229 \\
Hct $(\%)$ & $37.13 \pm 3.47$ & $37.62 \pm 3.07$ & $38.60 \pm 2.73$ & $\mathbf{0 . 0 4 2}$ \\
$\mathbf{M C V}(\mathbf{f L})$ & $79.12 \pm 6.06$ & $78.30 \pm 6.22$ & $80.02 \pm 3.64$ & 0.302 \\
$\mathbf{U I B C}(\boldsymbol{\mu m o l} / \mathbf{L})$ & $260.03 \pm 62.02$ & $249.89 \pm 77.43$ & $252.51 \pm 73.01$ & 0.739 \\
SI $(\boldsymbol{\mu m o l} / \mathbf{L})$ & $61.26 \pm 27.43$ & $69.13 \pm 34.40$ & $69.83 \pm 22.26$ & 0.217 \\
\hline
\end{tabular}

$\mathrm{Hb}=$ hemoglobin, $\mathrm{Hct}=$ hematocrit, $\mathrm{MCV}=$ mean corpuscular volume, $\mathrm{SI}=$ serum iron, $\mathrm{UIBC}=$ unsaturated iron binding capacity

impaired iron metabolism is the other effect of SDB. The published literature documented the increased incidence of anemia in patients underwent adenotonsillectomy $[3,12,13]$ and improved anemia after operations without any iron supplementation [11]. Unfortunately, there is no enough data to explain pathogenesis of impaired iron metabolism in these patients. There are two potential causes of impaired iron metabolism in SDB; nutritional iron deficiency and anemia of chronic disease secondary to chronic inflammation. Iron deficiency is the commonest nutritional deficiency worldwide according to WHO and affects a significant part of the population [23]. WHO estimates iron deficiency in $2.5 \%$ of pediatric population and describes an abnormality if the rate more than 5\% [24]. The evaluation iron deficiency starts with measurement of $\mathrm{Hb}$ and Hct levels that are non-specific markers. Ferritin, serum iron, total iron binding capacity or unsaturated iron binding capacity are widely used for differential diagnosis and confirmation of iron deficiency. The decreased $\mathrm{Hb}(<$ $11 \mathrm{~g} / \mathrm{dL})$ and ferritin $(<16 \mu \mathrm{g} / \mathrm{L})$ levels are important for diagnosis of iron deficiency anemia. Anemia of chronic disease is an iron metabolism disorder that might be associated with neoplastic diseases, chronic immunologic diseases, chronic inflammation and trauma [18]. The main mechanism of anemia of chronic disease is depending on increased levels of IL1, IL-6, TNF- $\alpha$ and CRP. These cytokines affect the erythropoietin synthesis from kidney that results with decreased erythropoiesis and decreased iron absorption from gastrointestinal system [25]. The increased releasing of Hepsidin in chronic inflammatory process plays an important role of decreased iron absorption from intestine. Patients have normochromic normocytic anemia in early stages that progress to hypochromic microcytic anemia. Ferritin levels might be normal or increased in these patients, which is an acute phase reactant. Unsaturated iron binding capacity or total iron binding capacity levels were decreased with serum iron. These findings are crucial to differentiate anemia of chronic disease and iron deficiency anemia.

The increased levels of TNF- $\alpha$, IL- 6 and CRP have been reported in patients with OSA [14-16]. The decreased IL-10 levels in non-obese children with OSA was documented that corrected after adenotonsillectomy [17]. IL-10 is an antiinflammatory cytokine which is negative correlated with OSA severity [26].

We documented anemia $(\mathrm{Hb}<11 \mathrm{~g} / \mathrm{dL})$ rate in $14 \%$ of those patients underwent adenotonsillectomy for SDB. This rate is much more than WHO expectations (2.5\%) which is abnormal. Hct, MCV, serum iron and unsaturated iron binding capacity were significantly decreased in patients with anemia. However, only 8 (33\%) patients had low ferritin levels in patients with anemia. Additional, 16 (67\%) of those patients with anemia had normal ferritin levels. The mean ferritin levels were $23.23 \pm 17.27$ and $21.27 \pm$ $15.44 \mu \mathrm{g} / \mathrm{L}$ in patients with anemia and non-anemic patients, respectively (Table 1). These findings are supporting the role of inflammatory process in anemia. Louise et al. [27] analyzed the association of ferritin with OSA in adults. They did not find an association between apnea-hypopnea index and ferritin levels but they documented the negative correlation between apnea-hypopnea index and ferritin levels in only women patients. The same study is indicating the association of lower minimum oxygen saturation and increased sleep stage shifts (that are correlated with severity of OSA) with increased ferritin levels. There are many publications supporting the chronic inflammatory process and increased ferritin levels in 
patients with OSA [28-31]. In our study, all patients with anemia had moderate or severe SDB clinical score (Table 3 ) but the difference was not significant. Everland et al. [11] reported the 57\% normal ferritin levels of those patients underwent adenotonsillectomy because of recurrent infection and upper airway obstruction. They indicated the role of chronic inflammatory process in these increased ferritin levels. Unfortunately, they did not do subgroup analysis addressing to the indication of adenotonsillectomy. We include only patients underwent adenotonsillectomy for SDB in the current study. On the other hand, Elverland et al. [11] reported the corrected $\mathrm{Hb}$ levels and iron metabolism at postoperative 6th month. This result is an important characteristic of anemia of chronic disease. Iron metabolism immediately becomes normal levels when you correct the underlying disease [25]. Chronic inflammation is the main cause of anemia in these patients that corrected after adenotonsillectomy.

Kerstein et al. [13] reviewed 94 children undergoing adenotonsillectomy. They categorized the study population according to age; Group A: 0-2 years, Group B: 2-6 years, Group C: above 6 years. They reported $18 \%$ anemia rate (17 patients) in this study population. Of those 17 patients, anemia rate was significantly increased in Group A. We report the increased anemia rate in children under 3 years of age that correlated with Kerstein et al. [13]. Although iron requirement decreases progressively after the sixth month of life until 3 years [25], the high rate of anemia in this age group SDB patients suggests that small patients may be more affected by inflammation. Bitar et al. [20] reported the increased SDB score in patients under 3 year-olds that indicates children under 3 yearolds were much affected than older children [20]. Children under 3 years of age might be influenced from SDB much more than older children according to our results. SDB clinical score, in the current study, was increased in children under 3 years of age that supports SDB severity in this cohort. Thirty out of 49 children less than 3 years of age had severe SDB clinical score (Table 2). Kerstein et al. [13] analyzed the results depending on the mean values of each of subgroups. They reported hypochromic $(\mathrm{MCH}<27$ $\mathrm{pg})$ and microcytic (MCV $<76 \mathrm{fL})$ mean values in children under 2 year-olds. The other subgroups had low-normal mean values. Unfortunately, they did not compare the mean values of 17 patients with anemia and non-anemic patients. It is not possible to clarify type of anemia without evaluation of anemic patients.
We found significantly decreased Hct, MCV, serum iron and unsaturated iron binding capacity levels in patients with anemia in comparison with non-anemic patients. Additional, only $8(33 \%)$ out of 24 patients had low ferritin levels (Table 1). These findings support an impaired iron metabolism secondary to inflammation.

\section{The Limitations of the Study}

This is a retrospective observational study which is the main limitation of paper. We did not have a control group. Additional, we were not able to analyze inflammatory markers owing to the retrospective setting. It would be better if we had inflammatory markers and cytokine levels. Polysomnography test is a gold standard test to evaluate SDB that supports objective results. Unfortunately, we do not use polysomnography as a routine test for evaluation of SDB; we use a questionnaire. We could have used subset analysis if we had polysomnography test results.

\section{Conclusions}

SDB is an important health problem in children that may result in many behaviors or growth problem. Unfortunately, there is no enough data about iron metabolism in pediatric SDB patients. Anemia in SDB might be result of iron deficiency or chronic inflammatory process.

\section{Conflict of interest}

The authors disclosed no conflict of interest during the preparation or publication of this manuscript.

\section{Financing}

The authors disclosed that they did not receive any grant during conduction or writing of this study.

\section{References}

[1] Lumeng JC, Chervin RD. Epidemiology of pediatric obstructive sleep apnea. Proc Am ThoracSoc 2008;15:242-52.

[2] Schlaud M, Urschitz MS, Urschitz-Duprat PM, Poets CF. The German study on sleep-disordered breathing in primary school children: epidemiological approach, representativeness of study sample, and preliminary screening results. Pediatr Perinat Epidemiol 2004;18:43140.

[3] Mira E, Benazzo M, Asti L, Marchi A, Spirano P, Losi R. Iron status in children undergoing tonsillectomy and its short-term modification following surgery. Acta Otolaryngol 1988;454:261-4.

[4] Lozoff B, Georgieff MK. Iron deficiency and brain development. 
Semin Pediatr Neurol 2006;13:158-65.

[5] Halterman JS, Kaczorowski JM, Aligne CA, Auinger P, Sziagyi PG. Iron deficiency and cognitive achievement among school-aged children and adolescents in the United States. Pediatrics 2001;107:1381-6.

[6] Angulo-Kinzler RM, Peirano P, Lin E, Garrido M, Lozoff B. Spontaneous motor activity in human infants with iron-deficiency anemia. Early Hum Dev 2002;66:67-79.

[7] Wintergerst ES, Maggini S, Hornig DH. Contribution of selected vitamins and trace elements to immune function. Ann Nutr Metab 2007;51:301-23.

[8] Oppenheimer SJ. Iron and its relation to immunity and infectious disease. J Nutr 2001;131:616-33.

[9] Zilberman M, Silverberg DS, Bits I, Steinbruch S, Wexler D, Sheps $\mathrm{D}$ et al. Improvement of anemia with erythropoietin and intravenous iron reduces sleep-related breathing disorders and improves daytime sleepiness in anemic patients with congestive heart failure. Am Heart J 2007; 154:870-6.

[10] Benz RL, Pressman MR, Hovick ET, Peterson DD. A preliminary study of the effects of correction of anemia with recombinant human erythropoietin therapy on sleep, sleep disorders, and daytime sleepiness in hemodialysis patients (The SLEEPO study). Am J Kidney Dis 1999;34:1089-95.

[11] Elverland HH, Aasand G, Miljeteig H, Ulvik RJ. Effects of tonsillectomy and adenoidectomy on hemoglobin and iron metabolism. Int J Pediatr Otorhinolaryngology 2004;68:419-23.

[12] Busuttil A, Kerr AI, Logan RW. Iron deficiency in children undergoing adenoid-tonsillectomy. J Laryngol Otol 1979;93:49-58.

[13] Kerstein R, Stimpson P, Caulfield H, Ellis G. Iron deficiency and sleep disordered breathing in children--cause or effect? Int J Pediatr Otorhinolaryngol 2009;73:275-80.

[14] Guilleminault C, Kirisoglu C, Ohayon MM. C-reactive protein and sleep-disordered breathing. Sleep 2004;27:1507-11.

[15] Barcelo A, Barbe F, Llompart E, Mayoralas LR, Ladaria A, Bosch $\mathrm{M}$, et al. Effects of obesity on C-reactive protein level and metabolic disturbances in male patients with obstructive sleep apnea. Am J Med 2004; $117: 118-21$.

[16] Ciftci TU, Kokturk O, Bukan N, Bilgihan A. The relationship be - tween serum cytokine levels with obesity and obstructive sleep apnea syndrome. Cytokine 2004;28:87-91.

[17] Gozal D, Serpero LD, Sans Capdevila O, Kheirandish-Gozal L.
Systemic inflammation in non-obese children with obstructive sleep apnea. Sleep Med 2008;62:489-94.

[18] Weiss G, Goodnough LT. Anemia of chronic disease. N Engl J Med 2005;352:1011-23.

[19] Sharifkashani S, Dabirmoghaddam P, Kheirkhah M, Hosseinzadehnik R. A new clinical scoring system for adenoid hypertrophy in children. Iran J Otorhinolaryngol 2015;27:55-61.

[20] Bitar MA, Rahi A, Khalifeh M, Madana LMS. A suggested clinical score to predict the severity of adenoid obstruction in children. Eur Arch Otorhinolaryngol 2006;263:924-8.

[21] Brodsky L. Modern assessment of tonsils and adenoids. Pediatr Clin North Am 1989;36:1551-69.

[22] Parikh SR, Coronel M, Lee JJ, Brown SM. Validation of a new grading system for endoscopic examination of adenoid hypertrophy. Otolaryngol Head Neck Surg 2006;135:684-7.

[23] Moy RJ. Prevalence, consequences and prevention of childhood nutritional iron deficiency: a child public health perspective. Clin Lab Haematol 2006;28:291-8.

[24] Iron deficiency anemia: assessment, prevention and control, A guide for program managers. (WHO web site) 2001. Available at: http://www.who.int/nutrition/publications/micronutrients/anaemia_iron_ deficiency/WHO_NHD_01.3/en/ Accessed January 13, 2017.

[25] Grandone A,Marzuillo P, Perrone L, Giudice EM. Iron metabolism dysregulation and cognitive dysfunction in pediatric obesty: Is there a connection? Nutrients 2015;7:9163-70.

[26] Dyugovskaya L, Lavie P, Lavie L. Lymphocyte activation as a possible measure of atherosclerotic risk in patients with sleep apnea. Ann N Y Acad Sci 2005;1051:340-50.

[27] O'Brien LM, Koo J, Fan L, Owusu JT, Chotinaiwattarakul W, Felt $\mathrm{BT}$, Chervin RD. Iron stores, periodic leg movements, and sleepiness in obstructive sleep apnea. J Clin Sleep Med 2009;5:525-31.

[28] Arnardottir ES, Mackiewicz M, Gislason T, Teff KL, Pack AI. Molecular signatures of obstructive sleep apnea in adults: a review and perspective. Sleep 2009;32:447-50.

[29] Kalantar-Zadeh K, Rodriguez RA, Humphreys MH. Association between serum ferritin and measures of inflammation, nutrition and iron in haemodialysis patients. Nephrol Dial Transplant 2004;19:141-9.

[30] Rambod M, Kovesdy CP, Kalantar-Zadeh K. Combined high serum ferritin and low iron saturation in hemodialysis patients: the role of inflammation. Clin J Am Soc Nephrol 2008;3:1691-701. 